\title{
Performance of Neighboring Indoor 5G Micro Operators with Dynamic TDD
}

\author{
Kimmo Hiltunen and Marja Matinmikko-Blue \\ Centre for Wireless Communications \\ University of Oulu \\ Oulu, Finland \\ kimmo.hiltunen@oulu.fi; marja.matinmikko@oulu.fi
}

\begin{abstract}
Local small cell deployments complementing the coverage of the existing outdoor networks are vital for the future 5G networks. To make the ultra-dense indoor network deployments more cost-efficient and to promote innovation and competition in the market, new local business and spectrum authorization models are needed. One such model is the recently proposed micro operator concept with spectrum micro licensing that allows the establishment of building-specific 5G networks. While evaluating the applicability of this new concept, the impact of the inter-operator interference on the performance of the victim micro operator needs to be understood. The system simulation results shown in this paper demonstrate how the cochannel interference between two uncoordinated micro operators utilizing dynamic TDD in the $3.5 \mathrm{GHz}$ band and located inside neighboring buildings can result in large throughput losses if the buildings are within a few hundred meters from each other. The main cause for these losses is the interference from the other operator's base stations. Finally, it is shown that the performance losses are highly scenario-specific: a denser victim network deployment, or a victim network with a higher load suffers less from any external interference. Therefore, the traditional approach of defining a single separation distance for the worst case scenario does not properly model the specifics of $5 \mathrm{G}$ networks and can lead to overly protective requirements.
\end{abstract}

Keywords-5G; micro operator; indoor; dynamic TDD; radio network performance; spectrum sharing

\section{INTRODUCTION}

Mobile communication networks have been traditionally deployed by mobile network operators (MNO) with countrywide exclusive licenses to provide wide area coverage. However, in case of $5 \mathrm{G}$ the importance of specific in-building networks will increase [1], in particular for the new trafficheavy services and use cases, and for deployments in the higher frequency bands. Instead of relying solely on the networks deployed by the MNOs, an emerging solution gaining increasing attention in research, regulation and industry is to allow other stakeholders, for example the venue owners, to deploy and operate their own in-building networks to satisfy local capacity and coverage needs [1].

A micro operator concept [2] with local spectrum micro licensing model [3][4] has recently been introduced for local $5 \mathrm{G}$ network deployments serving both its own restricted

This work in the "Micro-operator concept for boosting local service delivery in 5G (uO5G)" project was funded by Business Finland. customer set and acting as a neutral host for the customers of the overlying MNOs [5]. The introduction of a potentially large number of local indoor $5 \mathrm{G}$ micro operators calls for new local spectrum authorization models for novel deployment scenarios where different micro operators could deploy their local networks in neighboring buildings as discussed in [3]. For reliable service delivery, the micro operators would need to be issued local spectrum micro licenses. This calls for some form of interference coordination between the license holders and the potential incumbent spectrum users to guarantee that their operations remain free from harmful interference. In particular, this will be the case if the aim is to allow the micro operators to fully benefit from the flexibility offered by the dynamic time division duplex (TDD), which is an integral part of the $5 \mathrm{G} \mathrm{New}$ Radio [6]. For the development of such interference coordination mechanisms, interference characterization between the involved systems is critical. An initial analysis of the interference between adjacent micro operator deployments in the $3.5 \mathrm{GHz}$ band was presented in [3], where the minimum separation distance between two micro operators in adjacent buildings was analyzed. The work was continued in [7], where the propagation modeling was considerably enhanced and the impact of downlink inter-operator interference on the micro operator network performance was evaluated. This paper continues the work in [3] and [7] by further enhancing the inter-operator interference model to include also the impact of uplink interference on the performance of the victim network. Furthermore, the inter-operator interference between uplink and downlink is modeled as well. The goal is to evaluate both the impact of inter-operator interference and the required minimum separation distance between two neighboring micro operators sharing the same channel with $5 \mathrm{G}$ networks deployed inside different buildings.

The rest of this paper is organized as follows. Section II discusses the interference scenarios for an uncoordinated micro operator deployment with dynamic TDD. Section III introduces the system model, including the assumed network layout, propagation models, and models for the radio resource management and user performance. Then, a number of results evaluating the performance of the victim micro operator are presented and analyzed in Section IV. Finally, some conclusions are drawn and a few future research topics are discussed in Section V. 


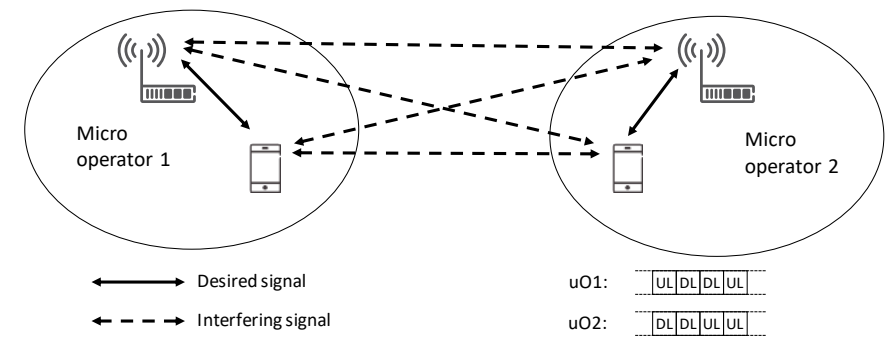

Fig. 1. Different inter-operator interference scenarios between neighboring micro operators.

\section{INTERFERENCE SCENARIOS}

Local micro operator deployments face various interference scenarios depending on the spectrum where they operate and operational characteristics of the networks. For example, neighboring cells can be either coordinated or uncoordinated. In case of dynamic TDD, a coordinated (or synchronized) deployment means that the neighboring cells have the same uplink:downlink (UL:DL) ratio and that the uplink slots in one cell are always aligned with the uplink slots in the other cells. Hence, there will never be time instants when the uplink transmissions in one cell will be interfered by downlink transmissions in other cells. However, that kind of interference scenarios are visible in uncoordinated (or unsynchronized) TDD deployments, for example when dynamic TDD with different UL:DL ratios have been applied in neighboring cells.

In all, four different inter-operator interference scenarios can be listed, as shown in Fig. 1:

- Interference from base station (BS) to mobile terminal (MT) (DL-to-DL interference), valid for both coordinated and uncoordinated TDD as well as for frequency division duplex (FDD).

- Interference from MT to BS (UL-to-UL interference), valid for both coordinated and uncoordinated TDD as well as for FDD.

- Interference from BS to BS (DL-to-UL interference), valid for uncoordinated TDD.

- Interference from MT to MT (UL-to-DL interference), valid for uncoordinated TDD.

Inter-operator interference towards downlink affects mostly the users, which are located closest to the interferers, e.g., the users located next to the outer walls of the buildings. However, in case of interference towards uplink, in particular without receive beamforming like in this paper, all users served by the same base station will be affected by the same level of interoperator interference.

\section{SYSTEM MODEL}

This section provides a brief description of the assumed network layout for a scenario with two neighboring micro operators. Furthermore, the applied propagation models, both for the indoor propagation within a building and for the building-to-building propagation between the different micro operators, are presented. Finally, models for radio resource

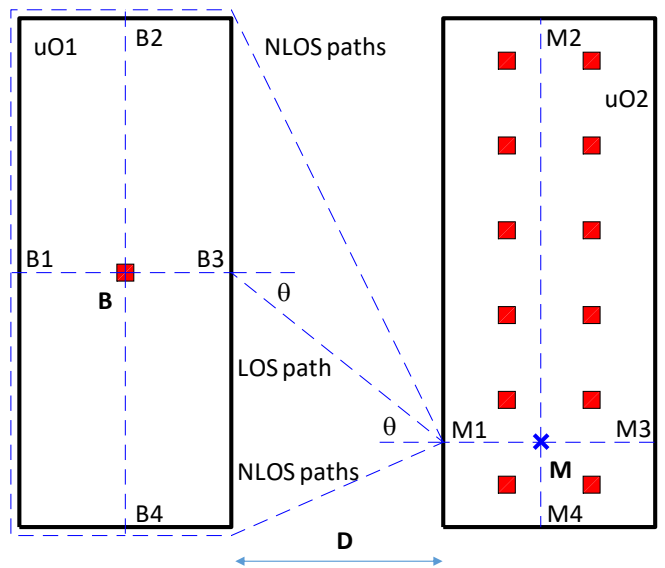

Fig. 2. Assumed network layout and the model for building-to-building propagation.

management and average user performance are discussed. A more detailed discussion of the assumed deployment scenario and the propagation modeling can be found in [8].

\section{A. Network Layout}

The evaluated network deployment consists of two equallysized buildings $(50 \times 120 \mathrm{~m}$, based on the indoor deployment scenario defined in [9]), located at a distance $D$ from each other, see Fig. 2. The buildings are assumed to be in line-ofsight (LOS) with each other and only one floor per building is modeled. Micro operator 1 (uO1) is assumed to be serving users within the first building, while micro operator $2(\mathrm{uO} 2)$ is serving users within the second building. Finally, it is assumed that $\mathrm{uO} 2$ has deployed 12 base stations per floor (with the same network layout as in [9]), while the density of base stations belonging to $\mathrm{uO} 1$ is varied during the evaluations.

Both networks are assumed to be operating in the $3.5 \mathrm{GHz}$ band and moreover, they are assumed to be operating on the same channel. Hence, the same center frequency $f_{c}$ equal to 3.5 $\mathrm{GHz}$ is assumed for both $\mathrm{uO} 1$ and $\mathrm{uO} 2$.

\section{B. Propagation Models}

In order to model the propagation both within a building and between the buildings, the coupling loss $C_{m, b}$ between mobile terminal $m$ and base station $b$ is calculated as

$$
C_{m, b, d B}=L_{m, b, d B}-G_{B S, m, b, d B}-G_{M T, m, b, d B}+X_{m, b, d B}
$$

where $G_{B S, m, b}$ and $G_{M T, m, b}$ are the base station and mobile terminal antenna gains, respectively, and $X_{m, b}$ is a log-normally distributed random value modeling the impact of shadow fading. Next, the assumed modeling of the path loss $L_{m, b}$ is discussed in more detail.

Path losses within a building are modeled using the $3 G P P$ Indoor - Mixed Office propagation model as defined in [9]. The path loss model includes both a LOS and a non-line-ofsight (NLOS) component, and the LOS probability is defined 
to decrease as a function of the distance between the base station and the mobile terminal. The standard deviation of the shadow fading is assumed to be equal to $3 \mathrm{~dB}$ (LOS) or $8 \mathrm{~dB}$ (NLOS) [9]. Furthermore, both the shadow fading and the LOS probability are spatially correlated, assuming correlation distances equal to $10 \mathrm{~m}$ or $6 \mathrm{~m}$ (shadow fading in LOS or NLOS), and $10 \mathrm{~m}$ (LOS probability) [9].

When it comes to modeling the path losses between the different buildings, four different sub-paths for each building, one through each building wall [10], are evaluated as shown in Fig. 2. Hence, for each link between a transmitter and a receiver, the total received power is calculated as a linear sum of the received powers from all the 16 different sub-paths. Each sub-path takes into account both the outdoor path loss between the outer wall reference points (B1-B4 and M1-M4 in Fig. 2), and the building penetration and indoor losses for both buildings. In all, the path loss per sub-path is calculated as

$$
L_{d B}\left(f_{c}\right)=L_{i n, 1}+L_{o w, 1}\left(f_{c}\right)+L_{o u t}\left(f_{c}\right)+L_{o w, 2}\left(f_{c}\right)+L_{i n, 2}
$$

In (2), $L_{i n}$ is the indoor loss, modeled as $L_{i n}=0.5 d_{2 D-i n}$, where $d_{2 D-i n}$ is the two-dimensional distance between the indoor node and the outer wall reference point [9]. Parameter $L_{o w}$ models the building wall loss and it consists of two components: one that depends on the angle of incidence $\theta$, when applicable, and the other that depends on the wall material and the center frequency $f_{c}$. Depending on the desired building penetration model (LOS or NLOS), $L_{o w}$ is calculated either as described in (3) [11] or in (4) [9].

$$
\begin{gathered}
L_{o w, L O S}=20(1-\cos \theta)^{2}+L_{\text {material }}\left(f_{c}\right) \\
L_{o w, N L O S}=5+L_{\text {material }}\left(f_{c}\right)
\end{gathered}
$$

Looking at Fig. 2, $L_{o w}$ is based on (3) for outer wall reference points B3 and M1, while it is based on (4) for all the other outer wall reference points. The model for $L_{\text {material }}\left(f_{c}\right)$ is taken from [9], and the applied $L_{\text {material }}$ value equal to $7.7 \mathrm{~dB}$ assumes that the penetrated building walls consist of $30 \%$ multi-pane windows and $70 \%$ concrete.

Parameter $L_{o u t}$ in (2) is the outdoor path loss between the outer wall reference points. In case of a LOS path (sub-path between B3 and M1), $L_{\text {out }}$ is based on a free space propagation model, and the applied distance is the sum of the outdoor and the indoor distances [10][12]. In case of NLOS paths, $L_{\text {out }}$ considers only the path between the outer wall reference points, and the path loss is based on the recursive microcell model [13], with a breakpoint for the path loss exponent at $300 \mathrm{~m}$. Finally, when it comes to the shadow fading model for the building-to-building propagation, standard deviation equal to 6 $\mathrm{dB}$ and correlation distance equal to $10 \mathrm{~m}$ are assumed.

\section{Radio Resource Management and User Performance}

The system model assumes that the cells within a micro operator's network are coordinated, while the cells belonging to different operators are uncoordinated. A random allocation of downlink and uplink slots is assumed for both networks, resulting in a 50:50 UL:DL ratio in average. A user is assumed to be connected to the closest base station belonging to the serving micro operator. Finally, a round-robin scheduler is assumed, either in time (DL) or in frequency domain (UL).

Downlink signal-to-interference-and-noise-ratio (SINR) for mobile terminal $m$, being served by base station $b$ on resource block $r$, is calculated as

$$
\begin{gathered}
\gamma_{m, b, r}=\frac{P_{b, r} C_{m, b}^{-1}}{I_{o w n, m, b, r}+I_{\text {other }, m, r}+I_{m t 2 m t, m, r}+N_{m, r}} \\
=\frac{P_{b, r} C_{m, b}^{-1}}{\sum_{\substack{j=1 \\
j \neq b}}^{B} \frac{\tau_{j, r} P_{j, r}}{C_{m, j}}+\sum_{k=1}^{K} \frac{\tau_{k, r} P_{k, r}}{C_{m, k}}+\sum_{s=1}^{S} \frac{\tau_{s, r} P_{M, s, r}}{C_{m, s}}+N_{m, r}}
\end{gathered}
$$

In (5), $I_{\text {own }}$ is the received power from all the other base stations belonging to the serving micro operator (having a total of $B$ base stations), while $I_{\text {other }}$ is the received power from all the base stations belonging to the other micro operators (a total of $K$ base stations). Finally, $I_{m t 2 m t}$ is the received inter-operator interfence from the mobile terminals belonging to the other micro operators. In case of the DL-to-DL interference scenario, $I_{\text {other }} \geq 0$, while $I_{m t 2 m t}=0$. In case of the UL-to-DL interference scenario the situation is the opposite: $I_{\text {other }}=0$ and $I_{m t 2 m t} \geq 0$. In more detail, parameter $P_{b, r}$ is the transmission power from base station $b$ on reference block $r, C_{m, b}$ is the coupling loss between mobile terminal $m$ and base station $b, N_{m, r}$ is the thermal noise power per resource block of mobile terminal $m$, and $\tau_{j, r}$ is an activity factor indicating whether transmitter $j$ is occupying resource block $r: \tau_{j, r}=1$, if $j$ is transmitting on resource block $r$, otherwise $\tau_{j, r}=0$.

The total transmission power $P_{M, m, b}$ for mobile terminal $m$, served by base station $b$ and occupying a total of $M_{m, b}$ resource blocks is defined as

$$
\begin{aligned}
& P_{M, m, b}=P_{0, b}+10 \log _{10}\left(M_{m, b}\right)+\alpha_{b} \cdot C_{m, b, d B}, \\
& P_{M \min } \leq P_{M, m, b} \leq P_{M \max }
\end{aligned}
$$

where $P_{0}$ is the target for the received uplink power per resource block and $\alpha$ is the path loss compensation factor.

Uplink SINR is calculated as

$$
\begin{aligned}
& \gamma_{m, b, r}=\frac{P_{M, m, b, r} C_{m, b}^{-1}}{I_{o w n, m, b, r}+I_{\text {other }, b, r}+I_{b s 2 b s, b, r}+N_{b, r}} \\
& =\frac{P_{M, m, b, r} C_{m, b}^{-1}}{\sum_{\substack{t=1 \\
t \neq m}}^{T} \frac{\tau_{t, r} P_{M, t, r}}{C_{t, b}}+\sum_{s=1}^{S} \frac{\tau_{s, r} P_{M, s, r}}{C_{s, b}}+\sum_{k=1}^{K} \frac{\tau_{k, r} P_{k, r}}{C_{k, b}}+N_{b, r}}
\end{aligned}
$$

where $I_{b s 2 b s}$ is the total inter-operator interference from base stations belonging to the other micro operators, and $N_{b, r}$ is the thermal noise power per resource block of base station $b$. In case of the UL-to-UL interference scenario, $I_{\text {other }} \geq 0$, while $I_{b s 2 b s}=0$. However, in case of the DL-to-UL interference scenario, $I_{\text {other }}=0$ and $I_{b s 2 b s} \geq 0$. 
TABLE I.

ASSUMED PARAMETER VALUES

\begin{tabular}{|l|l|}
\hline \multicolumn{1}{|c|}{ Parameter } & \multicolumn{1}{c|}{ Value } \\
\hline Center frequency & $3.5 \mathrm{GHz}$ \\
\hline Total number of resource blocks & 100 \\
\hline Bandwidth of a resource block & $180 \mathrm{kHz}$ \\
\hline Base station transmission power & $24 \mathrm{dBm}$ \\
\hline $\begin{array}{l}\text { Mobile terminal minimum and } \\
\text { maximum transmission power }\end{array}$ & $-40 \mathrm{dBm}$ (min), 23 dBm (max) \\
\hline Receiver noise figure & $12 \mathrm{~dB}$ (BS), 9 dB (MT) \\
\hline Base station antenna gain & $5 \mathrm{dBi}($ omnidirectional) \\
\hline Mobile terminal antenna gain & $0 \mathrm{dBi}($ omnidirectional) \\
\hline Maximum spectral efficiency & $\begin{array}{l}10.6 \mathrm{bbs} / \mathrm{Hz}(\mathrm{DL}, 64 \mathrm{QAM}, 2 \text { streams) } \\
5.3 \mathrm{bps} / \mathrm{Hz}(\mathrm{UL}, 64 \mathrm{QAM}, 1 \mathrm{stream})\end{array}$ \\
\hline Uplink power control target $\left(P_{0}\right)$ & $\begin{array}{l}-75 \mathrm{dBm}(1 \mathrm{BS}, 1 \mathrm{dBser}) \\
-70 \mathrm{dBm}(12 \mathrm{BS}, 1 \mathrm{dBser}, 50 \% \text { load) }\end{array}$ \\
\hline Path loss compensation factor & 0.8 \\
\hline
\end{tabular}

After the SINR values have been defined for the occupied resource blocks, the average SINR value for each active link is calculated as a logarithmic average of the SINR values per resource block. Finally, the average SINR is mapped to a corresponding average user throughput as

$$
R_{m, b}=\frac{0.8 \beta_{m, b} \cdot \min \left(R_{\text {max }}, \log _{2}\left(1+\frac{\bar{\gamma}_{m, b}}{1.6}\right)\right)}{u_{b}}
$$

In (8), it is assumed that the average overhead due to control channels and data retransmissions is equal to $20 \%$ and the margin due to receiver imperfections is equal to $2 \mathrm{~dB}$. The maximum spectral efficiency $R_{\max }$ is defined by the highest available modulation and coding rate, as well as the maximum number of parallel data streams for each link. The impact of scheduling on the average user throughput is taken into account via parameters $\beta_{m, b}$ and $u_{b}$ : for downlink with time domain scheduling $\beta_{m, b}$ is equal to the channel bandwidth, while $u_{b}$ is equal to the total number of users served by base station $b$. For uplink with frequency domain scheduling, $\beta_{m, b}$ is the bandwidth allocated to user $m$, while $u_{b}$ is equal to one.

\section{Evaluation Results}

This section presents and analyzes a number of evaluation results for the impact of inter-operator interference between two neighboring micro operators. The main simulation parameters assumed in this paper have been listed in Table I. For all the simulations it has been assumed that $\mathrm{uO} 1$ is the victim operator, while $\mathrm{uO} 2$ is the interfering operator. Furthermore, the $\mathrm{uO} 2$ network is assumed to consist of 12 base stations, and the number of active users is set so that the average network load is equal to $90 \%$.

It is worth noting that the uplink power control is assumed to be quite aggressive, aiming at a fairly high received power target $\left(P_{0}\right)$, while at the same time making sure that only a few
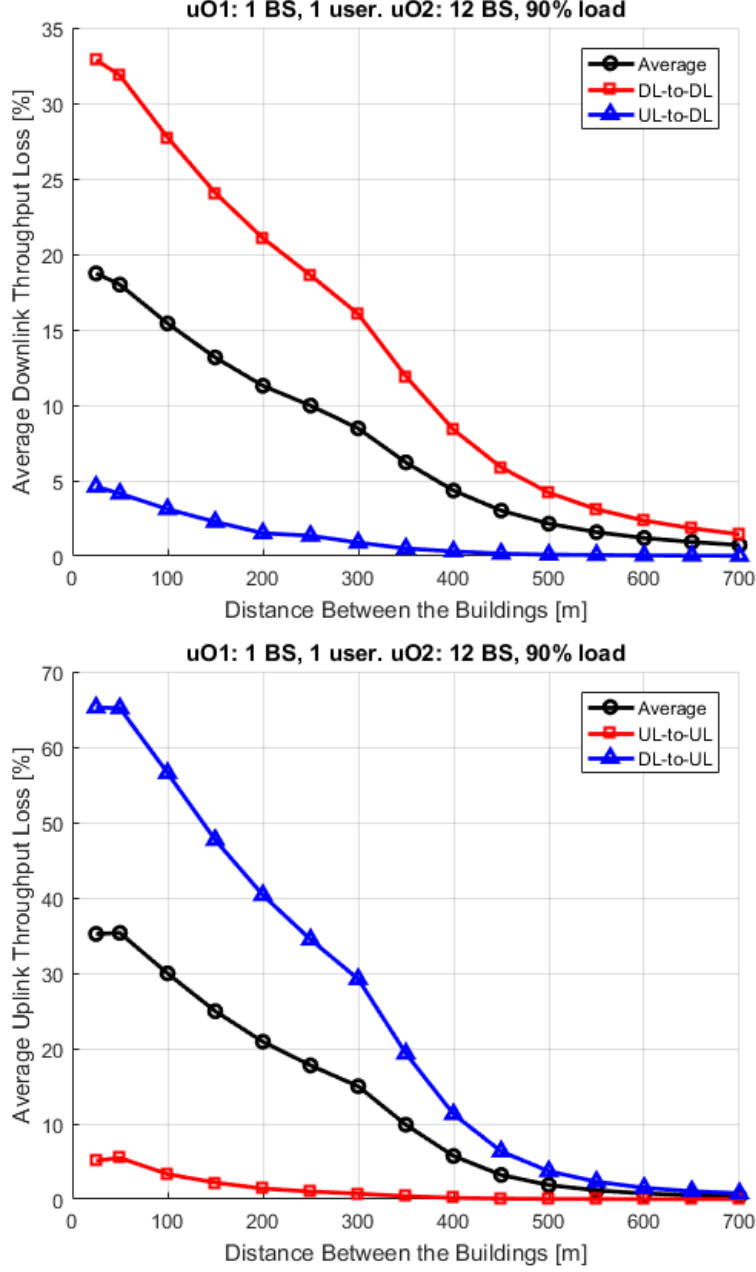

Fig. 3. Downlink and uplink throughput loss for micro operator 1 as a function of the distance between the buildings.

users are transmitting at the maximum power. When the target for the received power is high, many users experience an uplink SINR that exceeds the threshold for the maximum available bit rate. Therefore, the link becomes also quite tolerant of inter-operator interference; even though the uplink SINR is reduced as a result of $I_{\text {other }}$ or $I_{b s 2 b s}$, the corresponding impact on the actual user throughput may well be small. Due to the reduced link budget, denser network deployments allow the use of higher $P_{0}$ values. Similarly, when the network load is high, $M_{m, b}$ becomes small, and the value of $P_{0}$ can be increased to improve the protection against external interference.

The impact of inter-operator interference is measured by comparing the average system throughput for the multioperator scenario to the corresponding value for a single operator scenario. To start with, Fig. 3 presents the downlink and uplink throughput loss for a scenario where uO1 has deployed only one base station and the network is serving only one user. Three different curves are shown: throughput loss for the synchronized slots (DL-to-DL and UL-to-UL), throughput loss for the unsynchronized slots (UL-to-DL and DL-to-UL) and the average throughput loss (average of the above, taking the average UL:DL ratio into account). 


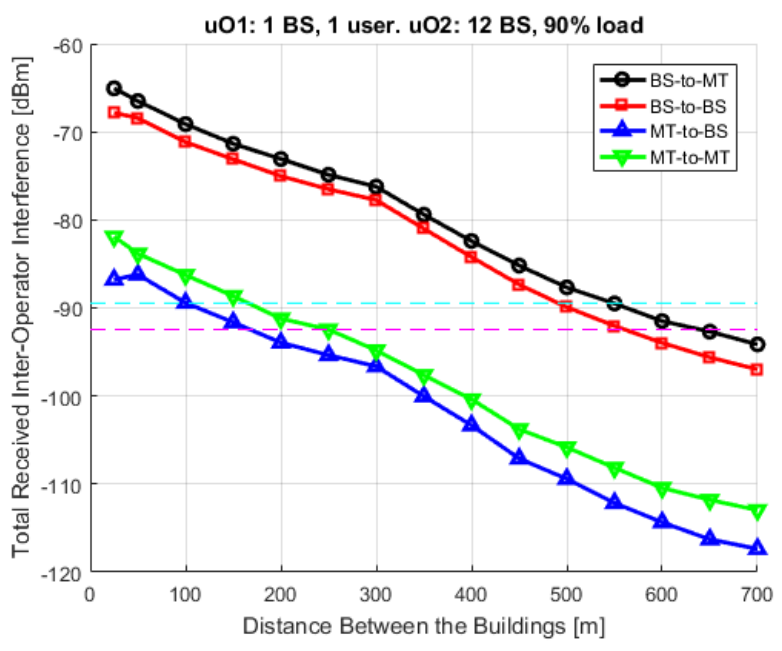

Fig. 4. $95^{\text {th }}$ percentile of the total received inter-operator interference power for all four interference scenarios between the two micro operators.

Looking at the obtained results, a few details can be highlighted. Firstly, the experienced throughput loss depends strongly on the distance $D$ between the buildings: both the level of the inter-operator interference (see the $95^{\text {th }}$ percentile curves in Fig. 4) and the corresponding performance losses are reduced as the distance $D$ is increased. For example, if a performance loss of up to a few percent is allowed, the distance between the buildings should be at least $500 \mathrm{~m}$. Secondly, it is very clear that both the downlink and the uplink suffer the highest performance loss during the slots, when the interoperator interference is caused by base stations. Looking at the curves in Fig. 4, this is by no means surprising: the $95^{\text {th }}$ percentiles of the received BS-to-MT and BS-to-BS interference levels are approximately $15-20 \mathrm{~dB}$ higher than the corresponding levels for the MT-to-BS and MT-to-MT interference. It is also worth highlighting, that when the distance between the buildings is less than $500-600 \mathrm{~m}$, the received worst case interference becomes higher than the receiver thermal noise floor $(-89 \mathrm{dBm}$ for the base station, -92 $\mathrm{dBm}$ for the mobile terminal as indicated by the dashed lines in Fig. 4). Finally, when the average downlink performance loss is compared to the average uplink performance loss, uplink seems to suffer more from the inter-operator interference, at least when the buildings are close to each other, i.e., with high levels of the inter-operator interference.

Fig. 5 presents the results for average downlink and uplink throughput loss as a function of the distance between the buildings. In order to evaluate the impact of both the victim network density and load on the experienced performance loss, a few different uO1 deployment scenarios are compared with each other: a) 1 BS \& 1 user, b) 12 BS \& 1 user, c) 12 BS \& $50 \%$ load, and d) 12 BS \& 90\% load. For all the evaluated deployment scenarios, the interfering $\mathrm{uO} 2$ network consists of 12 base stations and the average network load is equal to $90 \%$.

As can be noticed, the density of the victim network has a very large impact on the experienced performance loss, in particular for the downlink. This is due to the considerably higher received downlink carrier power levels seen in the denser network, resulting in higher SINR values. Due to the
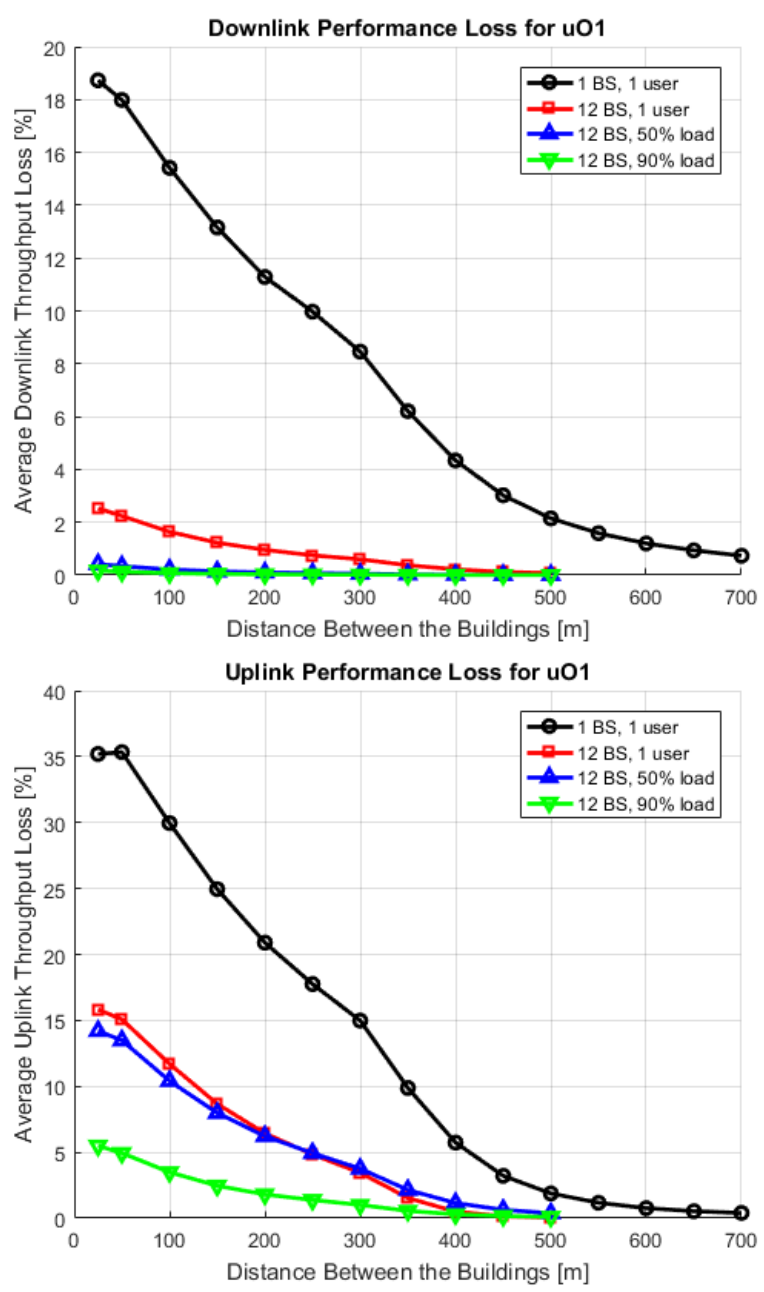

Fig. 5. Average downlink and uplink throughput loss for micro operator 1 as a function of the distance between the buildings, assuming a few different deployment scenarios.

very high link quality, also a high level of external interference is required before any noticeable reduction of the user performance can be detected. The situation is slightly different for the uplink, where the improved performance is not a result of the reduced link loss towards the serving base station, since with a constant $P_{0}$ the received carrier power would still stay the same. However, the reduced link losses enable the use of a higher $P_{0}$ value, resulting in a higher level of received carrier power, and hence, an increased protection against any external interference.

It becomes also clear that when the load of the victim network increases, the impact of the inter-operator interference becomes smaller. In downlink the reason is that the level of the intra-operator interference $\left(I_{\text {own }}\right)$ increases as a function of the network load, which means that the impact of the inter-operator interference $\left(I_{\text {other }}\right.$ or $\left.I_{m t 2 m t}\right)$ on the SINR becomes lower. In uplink, the main reason behind the improved performance is the increased $P_{0}$ at the very high network load (90\%) compared to the other cases.

The different victim network deployments can be compared also from the required minimum separation distance (MSD) 
TABLE II. MINIMUM SEPARATION DISTANCE

\begin{tabular}{|l|c|c|c|}
\hline \multirow{2}{*}{$\begin{array}{c}\text { Deployment } \\
\text { Scenario }\end{array}$} & \multicolumn{3}{|c|}{ Minimum Separation Distance (DL/UL) [m] } \\
\cline { 2 - 4 } 1 BS, 1 user & $638 / 571$ & $513 / 496$ & $382 / 415$ \\
\hline 12 BS, 1 user & $192 / 376$ & $70 / 338$ & $0 / 245$ \\
\hline 12 BS, 50\% & $0 / 415$ & $0 / 357$ & $0 / 248$ \\
\hline 12 BS, 90\% & $0 / 301$ & $0 / 185$ & $0 / 47$ \\
\hline
\end{tabular}

point of view. Here, MSD describes the minimum allowed distance between the buildings so that the observed average user performance is not degraded more than the desired threshold of $1 \%, 2 \%$, or $5 \%$.

Results for the MSD are summarized in Table II. As expected, the required downlink MSD is reduced together with an increased density and load of the victim network. The required uplink MSD is reduced as well, but there the main reason is not the reduced link loss or the increased network load, but the increased value of $P_{0}$ within the victim cells. One can also notice how the required MSD can be made smaller by allowing a higher loss of performance. Finally, while in the sparse victim network deployment the required MSDs are quite similar for both downlink and uplink, the dense network deployments are clearly limited by the uplink MSD, in particular for the higher network loads.

\section{CONCLUSIONS}

Local small cell deployments complementing the coverage of MNOs' outdoor networks have gained increasing attention in the development of the future 5G networks, in particular for high-traffic areas and for deployments within the higher frequency bands. In order to make such ultra-dense network deployments reality, new business and spectrum authorization models are urgently needed to open the mobile communication market. One such model is the recently introduced concept for $5 \mathrm{G}$ micro operators with local spectrum micro licensing where a key aspect is the interference between the micro operators, in particular when they are assumed to be sharing the same channel. The challenge is to define the required minimum separation distance between the neighboring micro operators so that the level of the received inter-operator interference is sufficiently low, yet making the spectrum usage efficient without having to resort to too conservative separation distances.

The impact of inter-operator interference on the performance of an indoor micro operator, as well as the required minimum separation distance between two uncoordinated micro operators utilizing dynamic TDD and sharing the same channel in the $3.5 \mathrm{GHz}$ band have been evaluated in this paper. The obtained results indicate that the interference received from the other operator's base stations is the main cause for both the downlink and uplink performance losses. In downlink, the impact of inter-operator interference, as well as the required minimum separation distance, is reduced for denser victim network densities (due to a higher received carrier power) and higher network loads (due to a higher level of intra-operator interference). Also in uplink a similar trend can be noticed, but there the improved victim network performance is caused by the increased received power target for the uplink power control. In all, due to the fact that the impact of the inter-operator interference is highly dependent on the deployment scenario, defining a single separation distance designed for the worst case situation can lead to overly protective protection distance requirements and less efficient use of spectrum.

Since the performance losses are mainly caused by the interference received from base stations, a natural topic for a future research would be to evaluate the potential performance gains, if the maximum transmission power of the interfering base stations would be reduced to protect the victim network. Furthermore, the performance analysis should be extended to cover also deployment scenarios within higher frequency bands, taking the impact of beamforming into account.

\section{REFERENCES}

[1] J. Zander, "Beyond the ultra-dense barrier: Paradigm shifts on the road beyond 1000x wireless capacity", IEEE Wireless Communications, vol. 24, no. 3, pp. 96-102, Jan. 2017.

[2] M. Matinmikko, M. Latva-aho, P. Ahokangas, S. Yrjölä, and T. Koivumäki, "Micro operators to boost local service delivery in 5G", Wireless Personal Communications, vol. 95, no. 1, pp. 69-82, Jul. 2017.

[3] M. Matinmikko, A. Roivainen, M. Latva-aho, and K. Hiltunen, "Interference study of micro licensing for $5 \mathrm{G}$ micro operator small cell deployments", in Proc. Conference on Cognitive Radio Oriented Wireless Networks and Communications (CrownCom), Lisboa, Portugal, Sep. 2017.

[4] M. Matinmikko, M. Latva-aho, P. Ahokangas, and V. Seppänen, "On regulations for 5G: Micro licensing for locally operated networks", Telecommunications Policy, in press.

[5] P. Ahokangas, M. Matinmikko, S. Yrjölä and I. Atkova, "Disruptive revenue models for future micro operator driven mobile business ecosystem", in Proc. The 24th Nordic Academy of Management Conference (NFF), Bodo, Norway, August 2017.

[6] S. Parkvall, E. Dahlman, A. Furuskär and M. Frenne, "NR: The new 5G radio access technology", IEEE Communications Standards Magazine, vol. 1, no. 4, pp. 24-30, Dec 2017.

[7] K. Hiltunen, M. Matinmikko-Blue, M. Latva-aho, "Impact of interference between neighboring 5G micro operators", Wireless Personal Communications, vol. 100, no. 1, pp. 127-144, May 2018.

[8] K. Hiltunen, M. Matinmikko-Blue, "Propagation model for evaluating the interference between neighboring indoor micro operators", accepted for publication at IEEE Vehicular Technology Conference (VTC) 2018 Spring.

[9] 3GPP, "Study on channel model for frequencies from $0.5 \mathrm{GHz}$ to 100 GHz”, 3GPP TR 38.901, V14.1.1, Jul 2017.

[10] E. Damasso, and L. M. Correia (ed.), "COST 231 Final Report: Digital mobile radio towards future generation systems", Tech. Rep., 1999.

[11] E. Semaan, F. Harrysson, A. Furuskär, and H. Asplund, "Outdoor-toindoor coverage in high frequency bands", in Proc. IEEE Globecom 2014 Workshop - Mobile Communications in Higher Frequency Bands, Austin, TX, USA, Dec. 2014.

[12] J.-E. Berg, "Building penetration loss along urban street microcells", in Proc. IEEE International Symposium on Personal, Indoor and Mobile Radio Communications (PIMRC), Taipei, Taiwan, Oct. 1996.

[13] J.-E. Berg, "A recursive method for street microcell path loss calculations", in Proc. IEEE International Symposium on Personal, Indoor and Mobile Radio Communications (PIMRC), Toronto, Canada, Sep. 1995. 